\title{
Advances in Dual Beam TEM Sample Preparation
}

\author{
M.V. Moore
}

FEI Company, 7451 NW Evergreen Parkway, Hillsboro, OR 97124

Today's samples present many challenges for transmission electron microscope (TEM) sample preparation. Focused ion beam (FIB) technology techniques continue to advance to provide solutions to meet the challenges due to new materials, structures of shrinking dimensions, and new TEM applications. While the FIB provides the advantages of site-specificity and wide material applicability to TEM sample preparation, a dual beam instrument with its added electron column gives expanded capabilities (Fig.1). The electron column adds higherresolution endpointing along with non-destructive imaging and surface protection of the sample (via electron-beam deposition); having coincidence of the ion and electron beams gives the operator immediate feedback about the sample being prepared.

The dual beam also enables the use of electron-beam-deposited Pt (from an organo-metallic precursor) for surface-sensitive samples. This type of deposition grows conformally over high aspect ratio structures and provides good contrast with many materials because of its high carbon content. This coating is useful, for example, in examining $\mathrm{Cu}$ seed layers for process development. Sub-100 nm-thick TEM membranes of $\mathrm{Cu}$ seed structures have been made automatically with recently enhanced automation software. Automated TEM sample preparation provides the advantage of reproducibility as well as increase in throughput to process development.[1]

Semiconductor design rules are approaching $100 \mathrm{~nm}$ (with gate lengths half that size); this increases the challenge in endpointing on these structures, centering ever-thinner TEM membranes on interconnects, and making thinner samples of increasing quality for high-resolution imaging. To improve the quality of TEM membranes for high-resolution work, low kV (Ga ion) beam cleaning can be used to reduce the amorphous layer of Si to enhance lattice imaging, as shown in Fig. 2.[2] The shrinking dimensions of structures to be cross-sectioned has stimulated further development of this technique. For sub-50 nm TEM cross-sections, low kV-assisted milling is necessary to avoid amorphizing the Si substrate during the milling process.

The relatively new application of TEM holography to dopant profiling of semiconductors presents demanding sample requirements. The sample must be very uniform in thickness (in the $200300 \mathrm{~nm}$ range), have an edge or hole in the same field of view as the area of interest (vacuum area for the reference beam), and be free from charging. Fig. 3 shows such a sample of a semiconductor transistor, that was successfully made in a dual beam using the high resolution of the ion beam for precision milling and the electron beam for endpointing and surface-quality monitoring.[3]

R.J. Young, Microsc. Microanal. 6 (Suppl. 2) (2000) 512.

[3] The author gratefully acknowledges assistance of FEI colleagues Sean Da, Jay Jordan, YC Wang and Richard Young, and collaboration with C. Kisielowski of LBL and S. Rozeveld and E.Beach of DOW Chemical. *SiLK is a trademark of the DOW Chemical Company. 


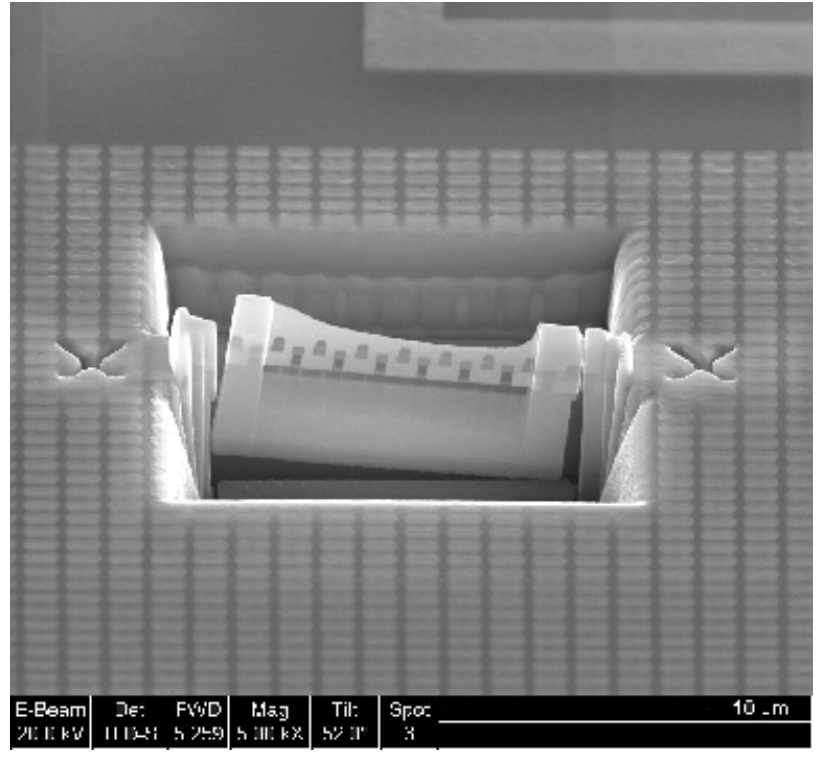

FIG. 1. Dual beam-prepared TEM sample of $\mathrm{Cu}$ damascene structure with SiLK*, a low-k dielectric. (Sample, courtesy IMEC. Image, S. Rozeveld and E. Beach)

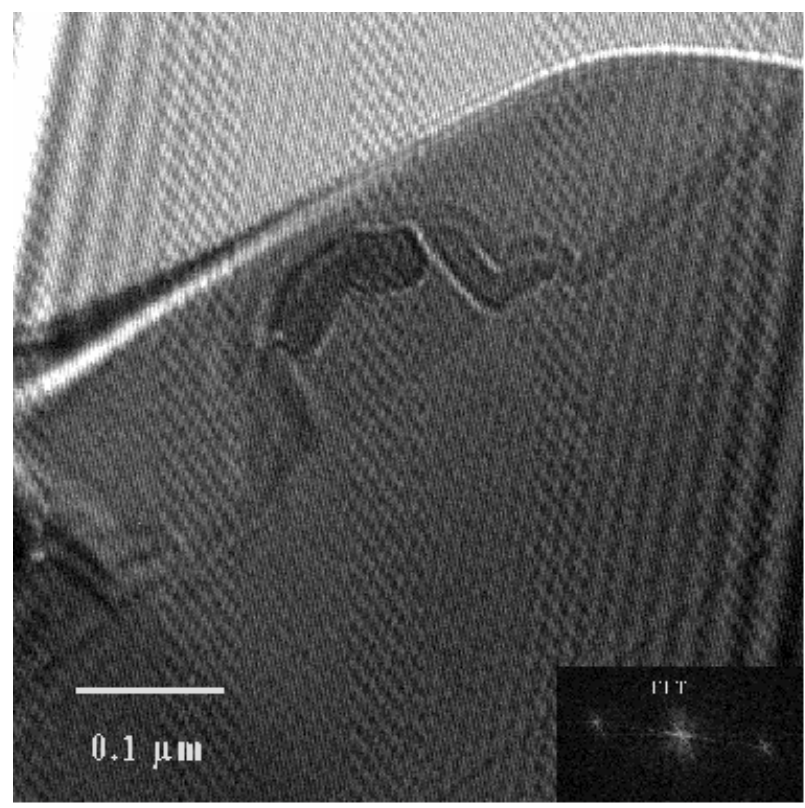

FIG. 3a. Hologram of semiconductor transistor.

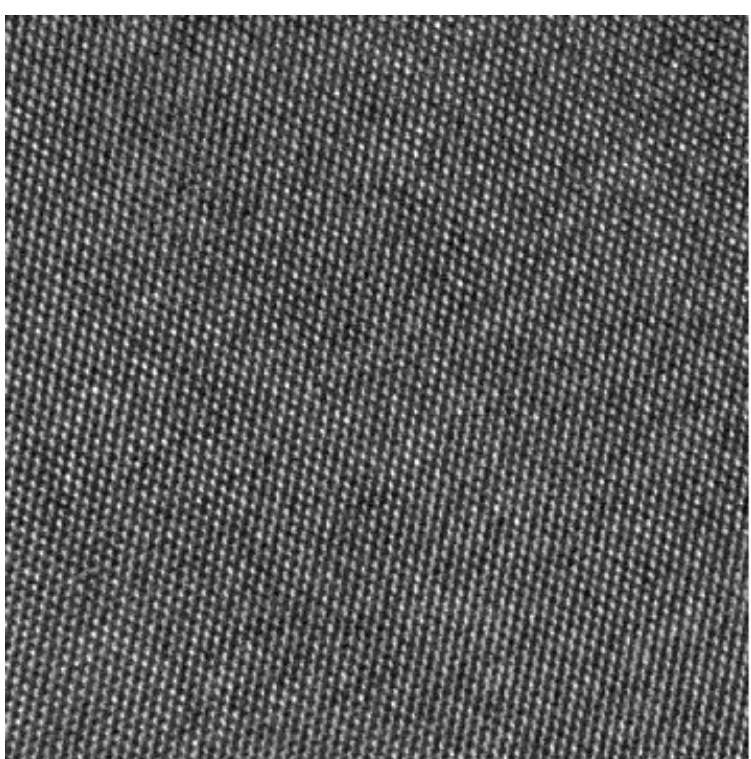

FIG. 2. Low kV Ga-ion cleaned Si lattice; image size is $20 \mathrm{~nm}$ (M.V.Moore, X. Xu, C. Kisielowski)

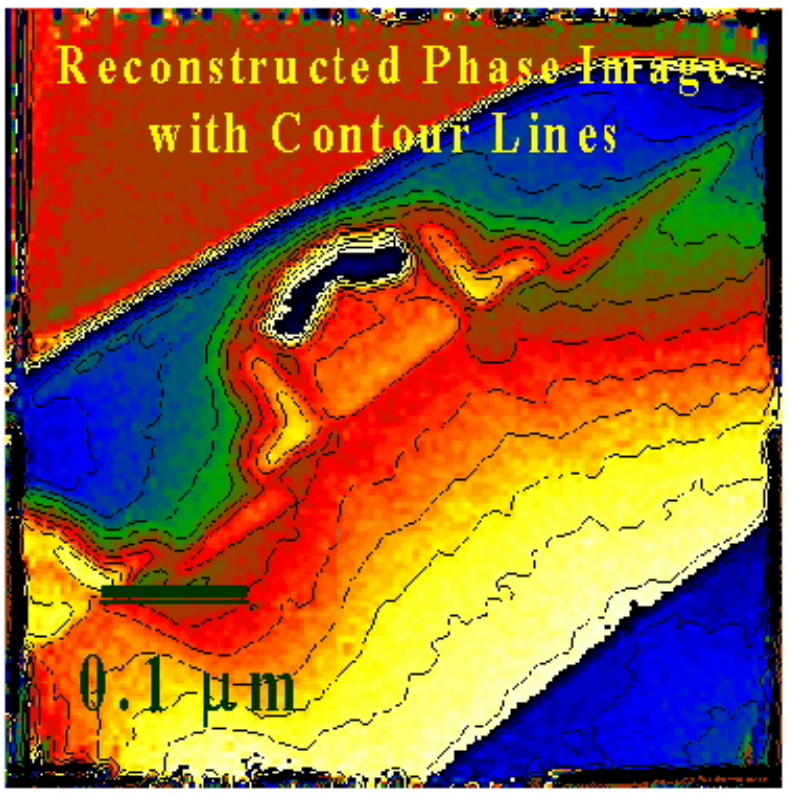

FIG. 3b. Reconstructed phase image of transistor region, useful for dopant profiling. 